\title{
ALGUNAS TENDENCIAS CURRICULARES EN LA FORMACIÓN DE EDUCADORES DE PÁRVULOS
}

La calidad de la formación docente en Chile ha surgido como un tema crítico en los últimos años (Ávalos, 2003), gatillado por los enormes desafíos para la educación en una sociedad de la información y globalizada, que a la vez funciona con significativas inequidades. La formación en Educación de Párvulos cobra especial relevancia en un contexto donde la cobertura ha aumentado significativamente, junto con la incorporación de la mujer al mundo laboral (Raczynski, 2006). Sumado a ello, gracias a los avances de la investigación en las neurociencias y la psicología, existe un creciente conocimiento sobre la importancia de la formación de los niños en los primeros años de vida como una etapa clave para el desarrollo cognitivo y emocional. Asimismo, las Bases Curriculares de la Educación Parvularia, promulgadas en 2001, le indican al país la amplitud y complejidad de aprendizajes que los niños y niñas pueden y deben lograr desde su nacimiento hasta los seis años de vida. En este escenario, la tradicional representación social de educadores o educadoras que cuida y entretiene a los niños no cumple con los desafíos requeridos (Moss, 2004). Por el contrario, se demanda una profesional que tome decisiones con fundamentos teóricos de acuerdo con cada contexto particular, que tenga competencias relativas al proceso de enseñanza-aprendizaje con grupos de niños diversos (social, cultural y cognitivamente), como también competencias en cuanto al uso de tecnologías de la información y comunicación, y al trabajo comunitario e intersectorial.

Sin embargo, la formación en Educación de Párvulos tiene significativas debilidades, lo cual ha implicado una preocupación tanto nacional (García-Huidobro, 2006) como internacional (Moss, 2004). En Chile, esta problemática cobra particular importancia considerando que en los inicios de los años 80 se desreguló el sistema de educación superior (Brunner, 2003). Este cambio abrió la posibilidad de formar educadores y educadoras en universidades 
privadas e institutos profesionales, sin grado de licenciatura en estos últimos. Como resultado, hoy existen 154 carreras de Educación de Párvulos a lo largo de todo el país, con una oferta sin regulaciones básicas que permitan orientar condiciones de funcionamiento mínimo curricular y organizacionalmente.

Frente a estas tensiones y a la vez desafíos de país, el Ministerio de Educación, Fundación INTEGRA y la Junta Nacional de Jardines Infantiles (JUNJI) encargaron a la Universidad Alberto Hurtado un estudio sobre la formación inicial de educadores y técnicos de educación parvularia. Los objetivos del estudio fueron: (i) catastrar y analizar la oferta de formación inicial en la educación superior y (ii) elaborar una propuesta curricular general, que especifique las competencias mínimas que debieran desarrollar en el marco de la educación superior los profesionales y técnicos de la educación parvularia en Chile.

Para el desarrollo del estudio se revisaron las propuestas curriculares de 95 carreras del país, se examinaron con mayor profundidad siete casos de estudio y se recogieron las principales demandas y visiones de distintos actores sociales (jefes de carreras, estudiantes, profesionales del Ministerio de Educación, JUNJi, INTEGRA y expertos en el área). Se analizaron, entre otros temas, las tendencias curriculares chilenas en la formación de estos educadores y se identificaron las principales tensiones y nudos críticos. A partir de ello, se examinaron las tendencias curriculares en otros países y se analizó de qué forma se resuelven estos nudos en otros contextos.

En este artículo se presenta parte del estudio internacional, relativo al análisis de las mallas y planes de estudio de nueve casos de estudio de seis países distintos, tomando en cuenta la duración de las carreras, los contenidos que se abordan, las especializaciones que se ofrecen y los enfoques innovadores que se proponen. En una primera sección se presenta una síntesis de los principales nudos críticos que surgieron desde el estudio nacional, lo cual orientó las preguntas centrales del estudio internacional. Luego se presentan las características y resultados del estudio y, finalmente, algunas conclusiones que intentan destacar aquellos aspectos más relevantes de acuerdo con el contexto chileno. 
Es necesario tener presente que el contexto en que se implementan cada una de las propuestas curriculares revisadas son realidades particulares, diferentes a la realidad chilena. Variados factores contextuales están presentes en esta formación, tales como las condiciones institucionales de las universidades, la estructuración de las carreras, el perfil de los estudiantes y el estatus social de la profesión en cada país. Estos elementos hacen que las propuestas curriculares y las prácticas institucionales cobren distintos significados y resultados de acuerdo con cada escenario social. Por consiguiente, el análisis de los enfoques internacionales no implica mirarlos como modelos a seguir, sino más bien entregan luces, abren preguntas y posibilitan pensar y enriquecer nuevas propuestas de acuerdo con el contexto particular chileno.

\section{Tensiones en la formación de los educadores de párvulos en Chile ${ }^{2}$}

De acuerdo con los resultados del estudio chileno, en términos generales se constata una oferta heterogénea en la formación de estos educadores, que carece de una propuesta curricular básica consensuada con un núcleo central de temas o disciplinas que estén en la base de las diversas propuestas de formación. Las mallas curriculares tienden a ser rígidas, centradas principalmente en las competencias específicas y prácticas de la profesión, con menor presencia de saberes teóricos y competencias generales que apunten a una formación más integral e interdisciplinaria. En cuanto al funcionamiento de las carreras, se observa en la mayoría de los casos, aunque con excepciones, condiciones precarias de gestión y organización institucional, sumado a un bajo porcentaje de profesores de planta. Asimismo, existe una escasa producción de investigación en educación infantil y generalmente un bajo nivel de especialización académica de los docentes.

2 Este apartado se desarrolla con profundidad en el estudio "Propuestas de mejoramiento para la formación de profesionales de educación parvularia”, Facultad de Educación, Universidad Alberto Hurtado 2008. Estudio encargado por el MINEDUC, JUNJI e INTEGRA. No publicado. 
Sobre la base del estudio de las mallas curriculares nacionales, destacan los siguientes nudos críticos:

- Las políticas públicas de los últimos años han priorizado ampliar la cobertura en las salas cunas ( 0 a 2 años). Sin embargo, las carreras tienden a orientar la formación en el segundo ciclo de la Educación Parvularia (3 a 6 años), mientras existe una débil formación y especialización en el primer ciclo ( 0 a 3 años). A pesar de que los temas vinculados con el cuidado de los infantes y/o los avances en el conocimiento de los primeros años de vida forman parte de varias carreras, ello no significa que los egresados tengan la preparación suficiente para trabajar con este grupo etario. En general, las prácticas laborales tienden a ser en niveles del segundo ciclo, sumado a que las carreras cuentan con pocos docentes especialistas en los primeros años de vida y desarrollan menos investigación en este campo.

Frente a esta situación, los debates actuales consideran distintas posibilidades, como especializaciones en un ciclo y una mayor formación interdisciplinaria (por ejemplo, con el área de salud), mientras otros insisten que la formación profesional debiera preparar habilidades para trabajar desde los 0 a 6 años, con un enfoque pedagógico y didáctico continuado y articulado, sin generar especialidades por edades. Por tanto, no hay consenso en este tema.

- La articulación entre la educación parvularia y básica es otro nudo crítico, pues se reconoce que estos profesionales poseen vacíos en términos disciplinarios para contribuir más activamente con dicha articulación. Sin embargo, no es claro que el método más efectivo para lograrlo sea especializarlos en conocimientos curriculares y disciplinares del primer ciclo básico (NB1). La especialización, de acuerdo con las mallas curriculares revisadas, descansa en un número de asignaturas y créditos insuficientes para lograr los dominios fundamentales de las didácticas curriculares del NB1. Además, se desdibuja su identidad profesional, pues su formación se ve subsumida por las lógicas formativas de la educación básica. Por ello, se abre la interrogante sobre el enfoque que debiera tomarse frente a esta temática: por un lado, es posible fortalecer la preparación disciplinar de las estudiantes; por otro, promover 
competencias del trabajo en equipo entre educadores de párvulos y de enseñanza básica; u otra posibilidad sería una especialización de posgrado.

- Existen altas demandas a la formación en cuanto a competencias para diseñar e implementar programas de trabajo con familias y comunidad. Esta es otra temática que pareciera necesitar más desarrollo curricular y un tratamiento más interdisciplinario, pues en general el trabajo con adultos y especialmente en contextos de pobreza es una debilidad de las carreras. Sin embargo, es debatible cuánto de ello es responsabilidad de la formación de pregrado y cuánto debería ser parte de la formación continua posterior de los profesionales. Además, implica cuestionar cuáles son los límites del campo de la Educación Parvularia y qué corresponde a profesionales e instituciones de otros sectores (por ejemplo salud, trabajo social, justicia). En ese sentido, más que habilidades para el trabajo comunitario sería necesario generar habilidades para el trabajo en redes e intersectorial.

- Asimismo, existen altas demandas, especialmente de parte de las instituciones INTEGRA y JUNJI, para una mayor formación en todo lo relacionado con la gestión y administración educativa. Nuevamente surge la interrogante de si esto debiera ser parte de la formación de pregrado o si corresponde a menciones o especializaciones posteriores de las profesionales.

- El alumnado de las carreras de Educación Parvularia presenta importantes carencias en cuanto a habilidades básicas, como expresión escrita y oral, comprensión lectora y razonamiento lógico. Además, comparativamente con otras carreras, se exigen bajos puntajes en la PSU y no se aprecian mecanismos complementarios de selección efectivos que midan o evalúen habilidades de entrada. Tampoco se observa que existan ofertas formativas durante la carrera que se aboquen explícitamente a desarrollar dichas deficiencias. Esta tensión invita a discutir sobre los requisitos de ingreso de los estudiantes, los mecanismos de selección y posibles propuestas curriculares que intenten revertir las carencias de entrada. 


\section{Descripción del estudio internacional}

Desde este análisis sobre los principales aspectos críticos de la formación de educadores de párvulos en Chile, se planteó realizar un estudio internacional con el fin de analizar las propuestas curriculares en otros países. Las preguntas que surgieron y que orientan el estudio fueron, por ejemplo, ¿cuáles son los requisitos de entrada de los estudiantes? ¿Se especializan por ciclos etarios? ¿Existen programas para la articulación con la educación básica? ¿Cómo se forman las competencias para trabajar con las familias y comunidad educativa? ¿Cómo se preparan para la gestión y administración de las instituciones educativas? ¿Cómo se trabajan las prácticas educativas y cómo se vinculan con la teoría?

El interés de este estudio fue revisar propuestas curriculares innovadoras que fueran un aporte a la calidad de la formación de estos educadores. Para la selección de países y universidades se utilizaron los siguientes criterios:

- Países y universidades con variedad de enfoques de formación inicial (por ejemplo, los países anglosajones se destacan por un enfoque cognitivo con énfasis en lenguaje y matemáticas, mientras que en los nórdicos ha prevalecido un enfoque pedagógico-social con énfasis en el desarrollo personal y social).

- Experiencias significativas e innovadoras para Chile de acuerdo con expertas consultadas. A este criterio le otorgamos gran relevancia.

- Universidades que tengan información accesible sobre las mallas curriculares. También implicó optar por las universidades que tenían su información en español o inglés.

- Se consideraron rankings internacionales de universidades (este criterio no siempre prevaleció pues se combinaron todos los criterios anteriores).

Los países seleccionados fueron Finlandia, Inglaterra, España, Nueva Zelanda y Australia. Además fue interés del equipo de investigación seleccionar al menos un país en vías de desarrollo de América Latina, entendiendo que puede enfrentar problemáticas y desafíos similares. Por ello, se decidió examinar universidades en Colombia, pues tiene más similitudes con Chile respecto de la 
discusión sobre formación inicial, el proceso de instalación de las reformas educacionales y las condiciones laborales y problemáticas que enfrentan los educadores. Además, Colombia tiene una interesante trayectoria de educación infantil no convencional y de programas de innovación como la Escuela Nueva.

Las universidades seleccionadas fueron las siguientes ${ }^{3}$ :

- University of Oulu, Finlandia.

- University of Helsinki, Finlandia.

- Greenwich University, Inglaterra.

- Universidad Complutense de Madrid, España.

- Auckland University, Nueva Zelanda.

- University of Melbourne, Australia.

- Western Sydney University, Australia.

- Universidad Pedagógica Nacional, Colombia.

- Universidad de Antioquia, Colombia.

La recopilación de datos se realizó centralmente por medio del análisis de documentación e información disponible en internet. Se usaron cuatro categorías generales para organizar la información: (1) títulos que se otorgan y duración de las carreras, (2) requisitos de admisión y egreso, (3) estructura general de las carreras y (4) cursos de las mallas curriculares. En cada caso, se establecieron las tendencias de cada malla según la frecuencia de aparición de un curso, área o temática.

Es importante mencionar que un estudio basado en el análisis de mallas curriculares tiene limitantes en cuanto no recoge información sobre la puesta en práctica de las propuestas, ni las perspectivas y opiniones de los actores sociales que las implementan.

\section{Resultados del estudio internacional}

A continuación se presentan los principales resultados del estudio. Las temáticas específicas que se analizan son las siguientes:

3 Otros países, tales como Israel, Cuba, Francia, Suecia y Dinamarca, fueron seleccionados pero no se revisaron en profundidad, pues no se encontró suficiente información o no había suficiente información en inglés o español. 
a. Títulos que se otorgan y duración de las carreras.

b. Requisitos de admisión y egreso.

c. Estructura general de las carreras.

d. Cursos de las mallas curriculares.

- Formación general.

- Ciencias de la educación.

- Formación específica de educadores de párvulos.

- Formación profesional general.

- Prácticas pedagógicas y actividades de titulación.

a) Títulos que se otorgan y duración de las carreras

Los estudios de pregrado analizados otorgan, en su mayoría, un título de Educador de Párvulos con el que los egresados pueden trabajar y/o realizar estudios de posgrado. Estos programas duran habitualmente tres años, con excepción de la Universidad de Melbourne, en Australia, y de las universidades en Colombia, en las cuales duran cuatro y cinco años respectivamente.

Otra modalidad que existe en algunas universidades, como Auckland University (Nueva Zelanda), Greenwich University (Inglaterra) y Western Sydney University (Australia), es ofrecer un programa de posgrado. Bajo esta modalidad, la carrera dura un año o un año y medio, y está dirigida solamente a quienes hayan terminado estudios de pregrado ${ }^{4}$. A su vez, en Greenwich University y Western Sydney University se ofrecen estudios de pregrado en educación e infancia, que forman para la investigación y para trabajar en programas de intervención relacionados con la infancia, otorgando una licenciatura (bachelor) en estudios de educación e infancia. Estas carreras no preparan para trabajar en el ámbito formal, pues aquello es una especialización que se entrega en posgrado. Así, se ofrece de manera separada la formación general de estudios en educación e infancia (nivel de pregrado) de la formación de educadores de párvulos para el trabajo directo con los niños/as (nivel de posgrado).

4 Los estudios de pregrado deben durar como mínimo tres años. En el caso australiano, se requieren estudios de pregrado en el área de la educación. En Inglaterra y Nueva Zelanda pueden ser en distintos campos de estudio. 
Tabla 1: Duración de las carreras de Educación de Párvularia y título que se otorga

\begin{tabular}{|l|l|l|}
\hline País & Duración & Título \\
\hline $\begin{array}{l}\text { Finlandia } \\
\text { Oulu University; } \\
\text { Helsinki University }\end{array}$ & 3 años & Kindergarten Teacher Education \\
\hline $\begin{array}{l}\text { Inglaterra } \\
\text { Greenwich University }\end{array}$ & $\begin{array}{l}1 \text { año (prerrequisito: grado de } \\
\text { licenciatura de mínimo 3 años de } \\
\text { estudios en educación u otra área) }\end{array}$ & Early Years Professional Status \\
\hline $\begin{array}{l}\text { España } \\
\text { Universidad Complutense }\end{array}$ & 3 años & $\begin{array}{l}\text { Diplomado en Maestro, } \\
\text { Especialidad en Educación } \\
\text { Infantil }\end{array}$ \\
\hline $\begin{array}{l}\text { Nueva Zelanda } \\
\text { Auckland University }\end{array}$ & 3 años & $\begin{array}{l}\text { Bachelor of Education Teaching } \\
\text { Early Childhood Education } \\
\text { Specialization }\end{array}$ \\
\cline { 2 - 4 } & $\begin{array}{l}1 \text { año (prerrequisito: grado de } \\
\text { licenciatura de mínimo 3 años de } \\
\text { estudios en educación u otra área) }\end{array}$ & $\begin{array}{l}\text { Graduate Diploma in Teaching } \\
\text { Early Years Childhood } \\
\text { Education }\end{array}$ \\
\hline $\begin{array}{l}\text { Australia } \\
\text { Melbourne University }\end{array}$ & 4 años & $\begin{array}{l}\text { Bachelor of Early Childhood } \\
\text { Education }\end{array}$ \\
\hline $\begin{array}{l}\text { Western Sydney } \\
\text { University }\end{array}$ & 3 años & $\begin{array}{l}\text { Bachelor of Early Childhood } \\
\text { Studies (Child and Family) }\end{array}$ \\
\cline { 2 - 4 } & $\begin{array}{l}1 \text { 1⁄2 año (prerrequisito: grado de } \\
\text { licenciatura de mínimo 3 años de } \\
\text { estudios en educación básica o en } \\
\text { estudios de infancia) }\end{array}$ & Master of Teaching \\
\hline $\begin{array}{l}\text { Colombia } \\
\text { Universidad Pedagógica } \\
\text { Nacional; Universidad de } \\
\text { Antioquia }\end{array}$ & 5 años & Licenciado en Pedagogía Infantil \\
\hline
\end{tabular}

Esta tendencia de carreras más cortas (uno a tres años) se instala en un esquema que promueve la realización de varios estudios de pregrado y posgrado (majors, minors, diplomas, magísteres). Por ejemplo, en el caso de Western Sydney University el pregrado en estudios de infancia dura tres años, y el grado para ser educador de párvulos dura un año y medio; por lo tanto, son cuatro años y medio en total para ejercer como educador. En Oulu University la carrera de implica cinco años de estudios, lo cual incluye el pregrado (tres años) y luego el magíster (dos años), como un continuo en la formación. Por lo tanto, el hecho de que las carreras sean más cortas no significa que las personas estudien menos años. Por el contrario, la propuesta es que las personas continúen formándose y especializándose.

En la mayoría de los países revisados se ofrece flexibilidad en la duración de las carreras, pues se puede optar por una modalidad 
de media jornada o jornada completa. Además, en las universidades de Australia e Inglaterra se reconoce la experiencia laboral de los estudiantes, lo cual acorta la duración de la carrera. Estas son formas de promover que las personas que trabajan con niños realicen estudios formales de grado profesional.

\section{b) Requisitos de admisión y egreso}

Los requisitos de entrada de los estudiantes universitarias son variables e incluyen currículum vitae, entrevista individual y/o grupal, cartas de referencia, ensayo escrito, condiciones médicas y antecedentes criminales. Se recopila información sobre niveles de motivación, experiencia previa en trabajo con niños, habilidades sociales, comunicacionales y de razonamiento matemático, y salud física y mental. Estos requisitos muestran una mayor gama de aspectos considerados en comparación con las universidades chilenas. Sin embargo, no se encontraron mayores detalles sobre los criterios de selección y los niveles de exigencia.

Tabla 2: Requisitos de admisión

\begin{tabular}{|l|l|}
\hline $\begin{array}{l}\text { Finlandia } \\
\text { Oulu University }\end{array}$ & $\begin{array}{l}\text { Currículum vitae (estudios, experiencia de trabajo con niños, servicio } \\
\text { a la comunidad), entrevista, situación en grupo, ensayo escrito. }\end{array}$ \\
\hline $\begin{array}{l}\text { Finlandia } \\
\text { Helsinki University }\end{array}$ & Notas de la escuela, carta de motivación, prueba de lenguaje. \\
\hline $\begin{array}{l}\text { Inglaterra } \\
\text { Greenwich University }\end{array}$ & $\begin{array}{l}\text { Estudios de licenciatura, prueba de término de la educación } \\
\text { secundaria (GCSE), habilidades comunicacionales, condiciones } \\
\text { médicas (físicas y mentales), antecedentes criminales. }\end{array}$ \\
\hline $\begin{array}{l}\text { Nueva Zelanda } \\
\text { Auckland University }\end{array}$ & $\begin{array}{l}\text { Cartas de recomendación, entrevista, prueba de lenguaje y } \\
\text { matemáticas, condiciones médicas (físicas y mentales), antecedentes } \\
\text { criminales. }\end{array}$ \\
\hline $\begin{array}{l}\text { Australia } \\
\text { Melbourne University }\end{array}$ & Prueba nacional de término de la educación secundaria. \\
\hline $\begin{array}{l}\text { Colombia } \\
\text { Universidad Pedagógica } \\
\text { Nacional }\end{array}$ & $\begin{array}{l}\text { Entrevista, examen pedagógico (Prueba de Potencialidad } \\
\text { Pedagógica), examen estandarizado (Icfes). }\end{array}$ \\
\hline
\end{tabular}

* No se encontró información disponible sobre los requisitos de ingreso en Western Sydney University, Universidad Complutense y Universidad de Antioquia. 
En el caso de Inglaterra, el gobierno ha generado un marco nacional de estándares ${ }^{5}$ de los educadores de párvulos. De esta forma se intenta crear una política nacional para regular la calidad de los adultos que trabajan con niños. Estos estándares son utilizados por las universidades con el fin de orientar las carreras y establecerlos como requerimientos de egreso, pues sólo con su cumplimiento se entrega el título profesional de "Early Years Professional Status".

\section{c) Estructura general de las carreras}

El análisis de las mallas curriculares se construye sobre la base de las categorías propuestas por García-Huidobro (2006) para clasificar los cursos, aunque se han realizado algunas adecuaciones. Las categorías utilizadas son: formación general, ciencias de la educación, formación específica de educadores de párvulos, formación profesional general y optativos.

- Formación general: cursos que proveen conocimientos y categorías de análisis para interpretar y orientarse en los contextos en los que vive. Por ejemplo: Políticas Educativas, Filosofía y Antropología Educativa, Cultura y Sociedad.

- Ciencias de la educación: cursos referidos a fundamentos y concepciones antropológicas y psicológicas relacionadas a las corrientes educativas y pedagógicas contemporáneas. Por ejemplo: Psicología del desarrollo, Bases pedagógicas, Teoría de la Educación.

5 Ver:www.cwdcouncil.org.uk/pdf/Early\%20Years/Draft_EYP_Standards_Aug_2006.pdf. Los estándares definidos son 39, agrupados en seis ámbitos. Primero, está el conocimiento de las necesidades de bienestar de los párvulos, sus características de desarrollo, sus diversas formas de aprendizaje, y su contexto social y cómo éste influye al niño. También se considera el conocimiento del currículum nacional y de las regulaciones legales sobre la infancia. Segundo, se definen estándares sobre la realización de "prácticas efectivas", lo cual se refiere a promover experiencias de desarrollo y aprendizaje por medio de la organización del ambiente y del tiempo, de la planificación y evaluación, y de la interacción cotidiana con los niños. Tercero, se espera que el adulto establezca interacciones positivas con los niños, de respeto, confianza, y apoyo, produciendo una comunicación receptiva, sensible y efectiva. Cuarto, el adulto debe practicar una comunicación positiva y de trabajo colaborativo con familias y cuidadores de los niños. Quinto, debe establecer un trabajo colaborativo con el equipo de trabajo, lo cual incluye un trabajo interdisciplinario con las personas dentro de la institución y de otros servicios. En último término, se espera que el adulto se ocupe de su desarrollo profesional continuo, lo que incluye habilidades básicas de lectura, razonamiento matemático y manejo tecnológico. Además se espera que tenga capacidad de generar reflexión crítica sobre su trabajo y de crear propuestas innovadoras. 
- Formación específica de educadores de párvulos: cursos que entregan una formación pedagógica con una aplicación más directa en la tarea educativa. Por ejemplo: Lenguaje y literatura infantil, Familia y comunidad, Didácticas y evaluación.

- Formación profesional general: cursos relativos a desarrollar competencias básicas de todo profesional, como segundo idioma, uso de TIC.

- Cursos optativos: buscan ampliar o profundizar la formación en temáticas de interés de los alumnos, estimulando una formación complementaria. Éstos se subdividen entre electivos de profundización, que corresponden a temas específicos de la carrera, y de formación general, que corresponden a cursos de otras carreras.

En esta sección analizamos la estructura global de las carreras, como el tiempo que se dedica a los cursos, el ordenamiento que se les da y la flexibilidad de las mallas de estudio. La tabla 3 muestra el tiempo que se dedica a los distintos tipos de cursos durante la carrera. En este análisis comparativo no se incluyen las prácticas pedagógicas y actividades de egreso, pues no se encontró suficiente información sobre el número de horas utilizadas en las prácticas de los estudiantes. 
Tabla 3: Tiempo porcentual que se le dedica a los cursos

\begin{tabular}{|c|c|c|c|c|c|}
\hline $\begin{array}{c}\text { Tipo de } \\
\text { cursos } \\
\text { País/ } \\
\text { Universidad }\end{array}$ & $\begin{array}{c}\text { Formación } \\
\text { general }\end{array}$ & $\begin{array}{c}\text { Ciencias de la } \\
\text { educación }\end{array}$ & $\begin{array}{c}\text { Formación de } \\
\text { educadores de } \\
\text { párvulos }\end{array}$ & $\begin{array}{c}\text { Formación } \\
\text { específica de } \\
\text { educadores de } \\
\text { párvulos }\end{array}$ & Optativos \\
\hline $\begin{array}{l}\text { Finlandia } \\
\text { Oulu University }\end{array}$ & $0 \%$ & $26 \%$ & $56 \%$ & $4 \%$ & $\begin{array}{l}14 \% \\
\text { (electivos de } \\
\text { formación } \\
\text { general) }\end{array}$ \\
\hline $\begin{array}{l}\text { Finlandia } \\
\text { Helsinki University }\end{array}$ & $0 \%$ & $10 \%$ & $60 \%$ & $12 \%$ & $\begin{array}{c}19 \% \\
\text { (electivos de } \\
\text { formación } \\
\text { general) }\end{array}$ \\
\hline $\begin{array}{l}\text { Inglaterra } \\
\text { Greenwich } \\
\text { University } \\
\end{array}$ & $0 \%$ & $0 \%$ & $100 \%$ & $0 \%$ & $0 \%$ \\
\hline $\begin{array}{l}\text { España } \\
\text { Universidad } \\
\text { Complutense }\end{array}$ & $0 \%$ & $19 \%$ & $65 \%$ & $0 \%$ & $\begin{array}{c}16 \% \\
\text { (electivos de } \\
\text { profundización) }\end{array}$ \\
\hline $\begin{array}{l}\text { Nueva Zelanda } \\
\text { Auckland } \\
\text { University }\end{array}$ & $0 \%$ & $20 \%$ & $75 \%$ & $0 \%$ & $\begin{array}{c}5 \% \\
\text { (electivos de } \\
\text { formación } \\
\text { general) }\end{array}$ \\
\hline $\begin{array}{l}\text { Australia } \\
\text { Melbourne } \\
\text { University }\end{array}$ & $3 \%$ & $3 \%$ & $94 \%$ & $0 \%$ & $0 \%$ \\
\hline $\begin{array}{l}\text { Australia } \\
\text { Western Sydney } \\
\text { University }\end{array}$ & $2.5 \%$ & $17.5 \%$ & $70 \%$ & $10 \%$ & $0 \%$ \\
\hline $\begin{array}{l}\text { Colombia } \\
\text { Universidad } \\
\text { Pedagógica } \\
\text { Nacional }\end{array}$ & $0 \%$ & $12 \%$ & $51 \%$ & $12 \%$ & $\begin{array}{l}24 \% \\
\text { (electivos de } \\
\text { profundización) }\end{array}$ \\
\hline $\begin{array}{l}\text { Colombia } \\
\text { Universidad de } \\
\text { Antioquia }\end{array}$ & $2 \%$ & $18 \%$ & $81 \%$ & $0 \%$ & $0 \%$ \\
\hline Promedio & $1 \%$ & $14 \%$ & $74 \%$ & $5 \%$ & $9 \%$ \\
\hline
\end{tabular}

La tendencia de las mallas curriculares es concentrar la mayor cantidad de tiempo en la formación específica. En promedio, el tiempo que se ocupa en estos cursos es de 74\%, lo que fluctúa desde 51 hasta $100 \%$. Estos se complementan con cursos de las ciencias de la educación, lo cual en promedio representan un 14\% de las mallas. Sin embargo, hay programas de estudio que incluyen escasos o ningún curso de estas materias, como Melbourne University y Greenwich University. 
Los cursos de formación general son casi nulos (en promedio $1 \%)$. Los de formación profesional general y los optativos también representan en promedio un bajo porcentaje del total (5 y 9\% respectivamente). No obstante, algunas carreras, como en el caso de Universidad Pedagógica Nacional y Helsinki University, dan importancia a la formación de competencias básicas de los estudiantes. También se observa que algunas universidades ofrecen cursos electivos, ya sea de formación general o de profundización.

Para analizar estas tendencias es importante considerar que la distribución de los cursos varía de acuerdo con la duración de las carreras y los requisitos de entrada de los estudiantes. En el caso de Inglaterra, por ejemplo, la carrera dura un año y es para personas licenciadas, por ello los cursos se concentran en un 100\% en las asignaturas específicas de educación de párvulos. En términos generales, no se encontró mayor información disponible sobre los perfiles de los estudiantes, sin embargo, es posible inferir que en los casos europeos y de Oceanía el perfil de ingreso es diferente a lo analizado para Chile. En varios casos las personas que ingresan ya son profesionales, por eso el tiempo dedicado a cursos de formación general y formación profesional es menor. Además, los itinerarios curriculares se construyen para un perfil de sujeto que continuará estudios de especialización o posgrado, por lo tanto, no es en una sola carrera donde se realizan los cursos generales sino en su recorrido en diversos estudios.

El orden de los cursos en las mallas curriculares es bastante variado, aunque, en general, los de formación general tienden a entregarse al comienzo, mientras las que asignaturas de formación específica se imparten durante toda la carrera, pero se concentran especialmente en el último año. Los cursos de ciencias de la educación se dan de forma mezclada presentándose en distintos momentos de la carrera. Por ejemplo, cursos como Sociología o Antropología de la Educación se ofrecen generalmente en primer o segundo año, mientras que el curso de Administración y Gestión Escolar se dicta en el último año de la carrera.

Las prácticas pedagógicas se dan en su mayoría durante varios semestres durante la carrera. En el caso de Melbourne University, 
Auckland University, Universidad Pedagógica Nacional y Universidad de Antioquia se imparten durante todos los semestres, como un curso continuo. En otras universidades, como en Helsinki University y Western Sydney University, se ofrece una práctica durante tres semestres. La Universidad Complutense es la única que solamente ofrece una práctica en el último semestre de la malla de estudios. Algunas carreras también terminan con una investigación-acción o tesis, generalmente junto a la última práctica, como en Melbourne University y las universidades de Colombia y de Finlandia. Aunque en algunas carreras no se menciona la realización de una tesis, como en el caso de la Universidad Complutense, Greenwich University y Auckland University.

Por último, las carreras estudiadas tienden a ofrecer una malla estructurada. De acuerdo con la información disponible, sólo algunas universidades ofrecen optativos y la mayoría no ofrece especializaciones (con excepción de la Universidad Pedagógica Nacional de Colombia). De ello se puede deducir que lo que da mayor flexibilidad a las carreras es su corta duración, por tanto mayor posibilidad de combinar con otros estudios y de especializarse por medio de minors, diplomados y/o magísteres.

\section{d) Cursos de las mallas curriculares}

d.1) Cursos de formación general

Como se mencionó, son escasos los que se imparten. Algunas carreras no los ofrecen y otras ofrecen máximo dos de estos cursos. Esto demuestra que la tendencia son carreras más cortas y con mayor nivel de especialización. A continuación se detallan los cursos de formación general impartidos.

Tabla 4: Cursos de formación general

\begin{tabular}{|l|l|}
\hline $\begin{array}{l}\text { Australia } \\
\text { Melbourne University }\end{array}$ & Estudios sobre Indígenas Australianos. \\
\hline $\begin{array}{l}\text { Australia } \\
\text { Western Sydney University }\end{array}$ & Psicología del Desarrollo 0-18 años; Ética y Temáticas Legales. \\
\hline $\begin{array}{l}\text { Colombia } \\
\text { Universidad de Antioquia }\end{array}$ & Seminario de Ética y Cultura \\
\hline
\end{tabular}




\section{d.2) Cursos de ciencias de la educación}

Los planes de estudio revisados ofrecen generalmente asignaturas relacionadas, tales como Psicología Educacional, Pedagogía, Sociología o Antropología de da Educación y Educación Especial. A continuación se presentan las asignaturas que se ofrecen.

Tabla 5: Cursos de ciencias de la educación

\begin{tabular}{|l|l|}
\hline $\begin{array}{l}\text { Finlandia } \\
\text { Oulu University }\end{array}$ & $\begin{array}{l}\text { Bases para la Educación y Docencia; Niños, Educación y Cultura; } \\
\text { Historia de la Infancia y Educación; Seminario de Revisión } \\
\text { Bibliográfica I-II; Psicología Educacional I-II; Pedagogía del } \\
\text { Aprendizaje; Bases de la Administración Social y Protección de los } \\
\text { Niños. }\end{array}$ \\
\hline $\begin{array}{l}\text { Finlandia } \\
\text { Helsinki University }\end{array}$ & $\begin{array}{l}\text { Bases Sociales y Culturales de la Educación; Perspectivas } \\
\text { Filosóficas, Históricas y Sociológicas en Educación. }\end{array}$ \\
\hline $\begin{array}{l}\text { Inglaterra } \\
\text { Greenwich University }\end{array}$ & No se imparten. \\
\hline $\begin{array}{l}\text { España } \\
\text { Universidad Complutense }\end{array}$ & $\begin{array}{l}\text { Sociología de la Educación; Didáctica General; Bases } \\
\text { Psicopedagógicas de la Educación Especial. }\end{array}$ \\
\hline $\begin{array}{l}\text { Nueva Zelanda } \\
\text { Auckland University }\end{array}$ & $\begin{array}{l}\text { Macro Influencias en Educación; Juego y Pedagogía; Diversidad y } \\
\text { Aprendizaje. }\end{array}$ \\
\hline $\begin{array}{l}\text { Australia } \\
\text { Melbourne University }\end{array}$ & Diferencias en el Desarrollo. \\
\hline $\begin{array}{l}\text { Australia } \\
\text { Western Sydney University }\end{array}$ & $\begin{array}{l}\text { Políticas y el Futuro de la Educación; Diversidad y Diferencias; } \\
\text { Juego, Desarrollo y Aprendizaje; Perspectivas Contemporáneas de } \\
\text { Infancia; Currículum y el Mundo Social; Ética en Educación. }\end{array}$ \\
\hline $\begin{array}{l}\text { Colombia } \\
\text { Universidad Pedagógica } \\
\text { Nacional }\end{array}$ & $\begin{array}{l}\text { Relación Cultura, Educación y Sociedad; Políticas, Constitución } \\
\text { Política de Colombia y Legislación; Historia y Epistemología de la } \\
\text { Pedagogía; Corrientes Pedagógicas Contemporáneas y su Incidencia } \\
\text { en Colombia. }\end{array}$ \\
\hline $\begin{array}{l}\text { Colombia } \\
\text { Universidad de Antioquia }\end{array}$ & $\begin{array}{l}\text { Epistemología e Historia de la Pedagogía; Nuevas Sociologías } \\
\text { de la Educación y Etnografía escolar; Sujeto y Educación I; } \\
\text { Corrientes Contemporáneas de Pedagogía y Didáctica; Antropología } \\
\text { Pedagógica y Formación Integral; Neuropsicología; Teoría y Gestión } \\
\text { del Currículo; Historia de la Infancia y la Adolescencia. }\end{array}$ \\
\hline
\end{tabular}

Los cursos de ciencias de la educación en los programas de estudios integran un enfoque interdisciplinario. Con el fin de comprender los procesos educativos se entremezclan distintas disciplinas como la Filosofía, Psicología, Biología, Antropología, Historia, Tecnología, además de la Pedagogía. En efecto, se puede observar que en varias mallas curriculares, como en la Oulu University, Helsinki University, Western Sydney University, se imparten asignaturas relacionadas con "estudios de educación e infancia", las 
cuales abordan una mirada interdisciplinaria. Es necesario mencionar que cursos relacionados con las neurociencias no aparecen como una temática preponderante, pues solamente se mencionan en las universidades de Colombia.

También parece interesante la propuesta de algunos programas de estudio de introducir una perspectiva de 'diversidad e inclusión', como en Auckland University, Western Sydney University y Melbourne University. En estos cursos se abordan temáticas ligadas a identidad social, etnias, género, clase social y discapacidad física/mental. Se trabaja desde un enfoque sociocultural de la infancia, poniendo en cuestionamiento nociones de "niño universal" y de "niño con déficit". De esta manera, se va más allá de cursos relacionados a "educación especial", y se introduce una perspectiva distinta desde las nociones de equidad, derecho de los niños, diversidad e inclusión.

En síntesis, existe una tendencia curricular de introducir variadas disciplinas para comprender, analizar y diseñar procesos pedagógicos, lo cual permite una propuesta de estudios amplia y enriquecedora. Esta perspectiva más holística es importante, especialmente considerando que tradicionalmente la psicología ha sido la disciplina predominante en el campo de la educación de párvulos.

\section{d.3) Cursos de formación específica en Educación Parvularia}

La mayoría de las mallas curriculares se concentran en asignaturas de formación específica de educación de párvulos. Entre estos cursos se trabajan temáticas relacionadas con Pedagogía Infantil, Currículum y Evaluación, Didácticas, Trabajo con Familia y Comunidad, Gestión y Administración Institucional, e Investigación. Debido a que estos cursos incluyen variados contenidos, el análisis en este apartado se subdivide por áreas temáticas.

\section{Pedagogía y didácticas}

En todos los programas de estudio se imparten asignaturas que se relacionan con currículum y trabajo pedagógico con párvulos. Se observa que estos cursos tienen énfasis en el aprendizaje, como también en el bienestar de los niños y el juego (ver Melbourne 
University y Auckland University). Además, existe una tendencia de abarcar un amplio espectro de contenidos y áreas del desarrollo/ aprendizaje, como áreas de lenguaje y comunicación, información y tecnologías de la comunicación y uso de multimedia, teatro, literatura, música, educación física, artes plásticas, ciencias del medio natural y social y formación socioafectiva. A continuación se detallan los cursos impartidos en estas áreas.

Tabla 6: Cursos de pedagogía infantil, currículum y didácticas

\begin{tabular}{|c|c|}
\hline $\begin{array}{l}\text { Finlandia } \\
\text { Oulu } \\
\text { University }\end{array}$ & $\begin{array}{l}\text { Bases Filosóficas y Científicas de la Educación Infantil; Bases Pedagógicas de la } \\
\text { Educación Infantil; Pedagogía de la Educación de Párvulos I-III, Educación Especial } \\
\text { Infantil; Multiculturalismo en Educación de Párvulos; Lenguaje, Comunicación y } \\
\text { Teatro; Idioma Extranjero y Multiculturalismo; Literatura, Arte y Teatro; Educación } \\
\text { Musical I-II; Educación Física; Educación Artística; Habilidades Manuales; Lenguaje } \\
\text { e Interacción; Ética y Educación Religiosa; Matemáticas y Ciencia; Educación } \\
\text { Medio Ambiental; Multimedia como un Proyecto de Aprendizaje; Información y } \\
\text { Comunicación Tecnológica como una Herramienta Pedagógica. }\end{array}$ \\
\hline $\begin{array}{l}\text { Finlandia } \\
\text { Helsinki } \\
\text { University }\end{array}$ & $\begin{array}{l}\text { Bases Psicológicas de la Infancia; Teorías del Desarrollo Infantil; Bases Pedagógicas } \\
\text { de la Educación Infantil; Planificación y Desarrollo en la Educación de Párvulos; } \\
\text { Aprendizaje en Acción; Educación Especial Infantil; Introducción a la Educación } \\
\text { de Párvulos y Educación Básica; Infancia y Procesos de Cambio; Educación y Salud; } \\
\text { Lenguaje e Interacción; Educación Matemática; Estudios del Medio Ambiente } \\
\text { Natural; Educación Religiosa y Moral; Educación Musical; Educación Física; } \\
\text { Educación Artística; Educación Manual; Literatura Infantil y Pedagogía Teatral. }\end{array}$ \\
\hline $\begin{array}{l}\text { Inglaterra } \\
\text { Greenwich } \\
\text { University }\end{array}$ & $\begin{array}{l}\text { Desarrollo Infantil y Aprendizaje: Desde el Nacimiento hasta los } 5 \text { años; Liderando } \\
\text { en Educación de Párvulos; Reconociendo Niños en Riesgo; Currículum y Evaluación } \\
\text { en Educación de Párvulos: Proyecto en un Jardín Infantil. }\end{array}$ \\
\hline $\begin{array}{l}\text { España } \\
\text { Universidad } \\
\text { Complutense }\end{array}$ & $\begin{array}{l}\text { Desarrollo de la Expresión Musical y su Didáctica; Desarrollo Psicomotor; } \\
\text { Adquisición y Desarrollo del Lenguaje I-II; Desarrollo del Pensamiento Matemático } \\
\text { y su Didáctica; Desarrollo de la Expresión Plástica y su Didáctica; Fundamentos } \\
\text { de la Lengua Española; Matemáticas; Conocimiento del Medio Natural Social y } \\
\text { Cultural; Nuevas Tecnologías Aplicadas a la Educación; Conocimiento del Medio } \\
\text { Natural; Literatura Infantil; Didáctica de las Matemáticas en Educación Infantil; } \\
\text { Artes Plásticas y Visuales y su Didáctica; Psicología de la Educación y del Desarrollo } \\
\text { en Edad Escolar. }\end{array}$ \\
\hline $\begin{array}{l}\text { Nueva } \\
\text { Zelanda } \\
\text { Auckland } \\
\text { University }\end{array}$ & $\begin{array}{l}\text { Bienestar en los Párvulos; Pedagogía para Párvulos; Enseñando, Aprendiendo, y } \\
\text { Evaluación en Educación de Párvulos; Mejorando los Logros de Aprendizaje en } \\
\text { los Párvulos; Movimiento en los Párvulos; Educación Tecnológica; Ciencia en la } \\
\text { Educación de Párvulos; Artes Visuales en los Párvulos; Música en los Párvulos; } \\
\text { Lenguaje y Literatura; Alfabetización-Múltiple (Multiliteracies); Matemáticas en } \\
\text { Párvulos; Danza y Teatro en Párvulos; Educación de Ciencias Sociales; Educación } \\
\text { en Aotearoa Nueva Zelanda. }\end{array}$ \\
\hline
\end{tabular}




\begin{tabular}{|c|c|}
\hline $\begin{array}{l}\text { Australia } \\
\text { Melbourne } \\
\text { University }\end{array}$ & $\begin{array}{l}\text { Aprendizaje y Enseñanza en Niños Pequeños; Aprendiendo, Enseñando, y Jugando } \\
\text { I-II; Desarrollo Físico; Desarrollo Cognitivo; Desarrollo Social y Emocional; Lenguaje } \\
\text { y Escritura/Lectura; Artes; Matemáticas; Salud y Educación Física; Sociedad y Medio } \\
\text { Ambiente; Información, Comunicación y Tecnología y Aprendizaje en los Niños; } \\
\text { Ciencia y Tecnología; Adaptando el Currículum I-II; Currículum Integrado. }\end{array}$ \\
\hline $\begin{array}{l}\text { Australia } \\
\text { Western } \\
\text { Sydney } \\
\text { University }\end{array}$ & $\begin{array}{l}\text { Problemáticas en los Contextos de la Educación de Párvulos; Patrones y Relaciones } \\
\text { Matemáticas; Niños, Ciencia y Tecnologías; Pensamiento Matemático de Párvulos; } \\
\text { Literatura Infantil; Currículum para Niños Menores de } 3 \text { Años; Convocando Niños } \\
\text { en el Currículum; Promoviendo Creatividad en el Aprendizaje de los Niños; } \\
\text { Matemáticas; Ciencia y Tecnología; Ambiente de Aprendizaje y Socialización } \\
\text { Positivos; Educación Aborigen. }\end{array}$ \\
\hline $\begin{array}{l}\text { Colombia } \\
\text { Universidad } \\
\text { Pedagógica } \\
\text { Nacional }\end{array}$ & $\begin{array}{l}\text { Seminario de Currículo Inicial; Articulación Inicial; Evaluación Inicial; Metodologías } \\
\text { de Trabajo Inicial; Introducción al Desarrollo Infantil; Desarrollo Socioafectivo; } \\
\text { Desarrollo Sociocognitivo; Desarrollo Biológico y Neurobiológico; Desarrollo, Salud } \\
\text { y Ecología Humana; Desarrollo Moral; Comunicación y Lenguaje I-II; Lúdica y } \\
\text { Psicomotricidad I-II; Arte I-II; Socialización I-II; Educación Matemática I-II; Ciencia } \\
\text { y Tecnología I-II, Enseñanza de una segunda lengua (Inglés). }\end{array}$ \\
\hline $\begin{array}{l}\text { Colombia } \\
\text { Universidad } \\
\text { de Antioquia }\end{array}$ & $\begin{array}{l}\text { Programas de Atención a la Infancia; Evaluación del Aprendizaje; Infancia y } \\
\text { Afectividad; Promoción y Prevención en Salud Infantil; Cognición y Desarrollo } \\
\text { Infantil; Integración Escolar en Pedagogía Infantil; Desarrollo Psicomotor; } \\
\text { Desarrollo Psicolingüístico; Desarrollo del Pensamiento Creativo; Procesos Orales, } \\
\text { de Lectura y Escritura; Fundamentación Lingüística; Literatura Infantil; Juego y } \\
\text { Arte en Educación Infantil; Expresión Musical y Danza; Pensamiento Matemático; } \\
\text { Didáctica de la Lectura y de la Escritura; Expresión Gráfico-Plástica; Historia; } \\
\text { Seminario Taller de construcción del Tiempo y Tiempo Histórico; Expresión } \\
\text { Teatral; Pedagogía del Cuerpo; Seminario de Educación Sexual; Seminario de } \\
\text { Actualización; Identificación y Caracterización de las Necesidades Educativas } \\
\text { Especiales; Adaptaciones Curriculares y de Intervención Pedagógica; Recursos } \\
\text { Didácticos Audiovisuales; Nuevas Tecnologías en Educación. }\end{array}$ \\
\hline
\end{tabular}

Desde el análisis de los cursos impartidos surgen algunas didácticas novedosas para la Educación Parvularia en Chile, como el aprendizaje de una segunda lengua (ver Oulu University, Western Sydney University, Universidad Pedagógica Nacional). Junto con ello, Oulu University, Auckland University y Western Sydney University introducen con fuerza temáticas relacionadas con multiculturalidad y con el aprendizaje de idiomas aborígenes. También aparecen asignaturas relacionadas con el área de la salud (ver Helsinki University, Western Sydney University, Melbourne University y Universidad de Antioquia). El enfoque de estos ramos incluye una mirada integral (especialmente en la Universidad de Western Sydney) incorporando aspectos como promoción de la salud, higiene, alimentación saludable, educación física y hábitos saludables de la familia. 
Asimismo, llama la atención el curso de "AlfabetizaciónMúltiple" (Multiliteracies) que se imparte en Auckland University. Se trabaja la interpretación y construcción de múltiples tipos de textos escritos, visuales y auditivos, como parte de los desafíos de una sociedad informática, globalizada y lingüísticamente diversa. Por último, la educación tecnológica y de medios de comunicación también surge como una temática relevante impartida en todas las universidades. Además, instituciones como Oulu University, Universidad Complutense y Universidad de Antioquia han enfatizado la importancia del uso de información tecnológica y medios audiovisuales como herramientas pedagógicas claves de estos educadores ${ }^{6}$.

La tendencia de las carreras analizadas es formar profesionales que tengan conocimiento de todas las áreas del aprendizaje, es decir, que puedan trabajar de forma holística e integrada las distintas áreas y didácticas. Incluso en Melbourne University y Universidad de Antioquia se imparten cursos de "Integración curricular", que tratan justamente de generar metodologías pedagógicas que integren las distintas áreas de aprendizaje y, a la vez, que prioricen las más significativas de acuerdo con el contexto sociocultural y las necesidades de los niños. Por lo tanto, los estudiantes no se especializan en una didáctica. Aunque en dos universidades se ofrecen especializaciones en ciertas materias, ello se realiza después de una formación general en las distintas áreas del aprendizaje. En la Universidad de Melbourne, luego del término de la carrera, se ofrecen diplomas (de un año) en: Artes, Sistemas Informáticos, Lenguajes Modernos y Música. Por otro lado, en la Universidad Pedagógica Nacional de Colombia, en el cuarto y quinto año, los estudiantes pueden "profundizar" en Inglés, Arte, Tecnología o en escenarios no convencionales.

En relación con la etapa etaria en que se forman los estudiantes, los cursos de formación tienden a prepararlos para trabajar con niños desde su nacimiento hasta 5/8 años (ver tabla $N^{0} 7$ ). La propuesta de extender la formación de párvulos hasta los ocho años pareciera

6 En Chile la educación tecnológica y de medios audiovisuales se ha incorporado especialmente en la formación de profesores básicos, como también en profesores de media. Sin embargo, esta es una temática menos abordada en la formación de estos educadores. 
interesante, a pesar de que los educadores habitualmente trabajan con niños hasta los 5 o 6 años. Esta extensión puede ser un intento de dar mayor articulación con la educación básica y de preparar a los profesionales para trabajar en niveles heterogéneos. Asimismo, puede significar un enfoque que sugiere que el cambio más significativo en el desarrollo de los niños se produce a los ocho y no a los seis años. También, entendiendo que los niños no se desarrollan de forma homogénea, por lo tanto permite que los educadores estén preparados para trabajar con niños con distintos niveles de desarrollo y aprendizaje, pudiendo desafiarlos de acuerdo con sus necesidades y potencialidades.

Esta tendencia muestra además una propuesta de formar una experticia pedagógica en el ciclo completo de educación de párvulos. En Melbourne University y Universidad Pedagógica Nacional de Colombia existe una especialización por edades en niños párvulos (0-5 años) y en niños de primer ciclo básico (5-8 años). Igualmente, los estudiantes de estas universidades están preparados para trabajar con niños de todo el rango etario (nacimiento - ocho años). También, la división entre ciclos se realiza entre el de párvulos y el de educación básica, y no entre el primer (0-3) y segundo ciclo (3-6) de la educación de párvulos.

Tabla 7: Formación según etapa etaria

\begin{tabular}{|l|l|}
\hline Finlandia & Niños de 0-8 \\
\hline $\begin{array}{l}\text { Inglaterra } \\
\text { Greenwich University }\end{array}$ & Niños de 0-5 \\
\hline $\begin{array}{l}\text { España } \\
\text { Universidad Complutense }\end{array}$ & Niños de 0-6 \\
\hline $\begin{array}{l}\text { Nueva Zelanda } \\
\text { Auckland University }\end{array}$ & Niños de 0-5 \\
\hline $\begin{array}{l}\text { Australia } \\
\text { University of Melbourne }\end{array}$ & Niños de 0-8 \\
\hline $\begin{array}{l}\text { Australia } \\
\text { University of Western Sydney }\end{array}$ & Niños de 0-8 \\
\hline $\begin{array}{l}\text { Colombia } \\
\text { Universidad Pedagógica Nacional }\end{array}$ & Niños de 0-8. \\
\hline $\begin{array}{l}\text { Colombia } \\
\text { Universidad de Antioquia }\end{array}$ & Niños de 0-7 \\
\hline
\end{tabular}


En síntesis, los programas de estudios analizados preparan a los estudiantes para ser expertos en todas las áreas de aprendizaje y generar un currículo integral. Esta formación general puede ser complementada con la especialización posterior en algunas materias. Además, se les prepara para trabajar con niños de todo el ciclo (0-5/8 años). Sobre esta base, en algunos casos se ofrecen especializaciones en educación de párvulos y en primer ciclo de educación básica.

Trabajo con familia y comunidad educativa

Uno de los aspectos más novedosos es que el enfoque en varios países es de un trabajo colaborativo con adultos en general. Esto incluye trabajo tanto con padres como con colegas de la misma institución y profesionales de otras instituciones y disciplinas (por ejemplo, salud $\mathrm{y}$ trabajo social). Esta perspectiva ha sido enfatizada especialmente en países como Finlandia e Inglaterra, donde además se ha fomentado la contratación interdisciplinaria de profesionales/técnicos en los centros educativos. De esta forma, el educador de párvulos no es un experto en trabajo con familia y servicio comunitario, sino que, más bien, debe saber cómo trabajar en red con otros profesionales e instituciones.

Por otra parte, la propuesta de la Western Sydney University parece interesante pues, además de enfatizar el trabajo coordinado con otros profesionales y servicios de la infancia, imparte asignaturas relacionadas con trabajo comunitario, apoyo a familias con niños con necesidades especiales y apoyo a familias en el cuidado infantil y salud familiar.

Es igualmente importante señalar que hay carreras que no incluyen asignaturas relacionadas con el tema de trabajo con familia, como en las universidades de Inglaterra, España y Nueva Zelanda. 
Tabla 8: Cursos de trabajo con familia y comunidad

\begin{tabular}{|l|l|}
\hline $\begin{array}{l}\text { Finlandia } \\
\text { Oulu University }\end{array}$ & Colaboración Educación de Párvulos. \\
\hline $\begin{array}{l}\text { Finlandia } \\
\text { Helsinki University }\end{array}$ & $\begin{array}{l}\text { Comunidades, Espíritu Social e Interacción } \\
\text { Social; Colaboración en instituciones de } \\
\text { Educación de Párvulos. }\end{array}$ \\
\hline $\begin{array}{l}\text { Inglaterra } \\
\text { Greenwich University }\end{array}$ & No se imparten. \\
\hline $\begin{array}{l}\text { España } \\
\text { Universidad Complutense }\end{array}$ & No se imparten. \\
\hline $\begin{array}{l}\text { Nueva Zelanda } \\
\text { Auckland University }\end{array}$ & No se imparten. \\
\hline $\begin{array}{l}\text { Australia } \\
\text { Melbourne University }\end{array}$ & $\begin{array}{l}\text { Relaciones Colaborativas; Familia y } \\
\text { Comunidad en una Sociedad Global. }\end{array}$ \\
\hline Australia & $\begin{array}{l}\text { Relaciones Colaborativas; Introducción a } \\
\text { Servicios para la Infancia; Aprendiendo por } \\
\text { Western Sydney University }\end{array}$ \\
$\begin{array}{l}\text { Medio de Servicios Comunitarios; Intervención } \\
\text { Temprana y Prevención en Contextos } \\
\text { Educacionales; Niños con Necesidades } \\
\text { Adicionales y sus Familias; Cuidado y Salud } \\
\text { Familiar: Niños y Adolescentes. }\end{array}$ \\
\hline $\begin{array}{l}\text { Colombia } \\
\text { Universidad Pedagógica Nacional }\end{array}$ & Familia, Escuela y Contexto \\
\hline $\begin{array}{l}\text { Colombia } \\
\text { Universidad de Antioquia }\end{array}$ & $\begin{array}{l}\text { Métodos de Trabajo con Familia y Comunidad, } \\
\text { Legislación Educativa y Familiar }\end{array}$ \\
\hline
\end{tabular}

\section{Gestión y administración institucional}

La formación en gestión y administración de instituciones educativas no tiene una preponderancia en los programas de estudio revisados. De las nueve universidades, solamente tres ofrecen una asignatura relacionada con este tema; estas son la Universidad Complutense, Western Sydney University y Universidad Pedagógica Nacional. Ellas ofrecen cursos generalmente introductorios al tema, en los que incluyen variados enfoques y temáticas relacionadas con gestión organizacional, trabajo interdisciplinario, aspectos legales, economía, entre otros. En algunos casos se abordan estas temáticas con mayor profundidad en estudios posteriores, como en el caso de Finlandia en que es una asignatura que se realiza en el magíster.

En conclusión, se observa que la gestión institucional es un contenido que se trabaja de forma introductoria o que no se imparte en las carreras analizadas. Probablemente sea un tema que 
se aborde con mayor profundidad en otros cursos de especialización en posgrado.

Tabla 9: Cursos de gestión y administración institucional

\begin{tabular}{|l|l|}
\hline España & Organización del Centro Escolar \\
Universidad Complutense & Servicios para la Infancia: Gestión y \\
\hline Australia & Administración \\
\hline Western Sydney University & Economía y Gestión de la Educación \\
\hline Colombia & \\
\hline Universidad Pedagógica Nacional & \\
\hline
\end{tabular}

Desarrollo profesional

Algunas universidades (ver tabla 10) ofrecen cursos vinculados con el "desarrollo profesional". Estas asignaturas incluyen diversas temáticas como: identidad profesional, trabajo interdisciplinario, ética profesional, inserción laboral, leyes y regulaciones laborales. También en algunos de estos cursos se mencionan contenidos valóricos y/o sociales, como es el desarrollo sustentable, participación y ciudadanía y equidad. Estos contenidos sugieren la importancia de trabajar competencias transversales -que incluyen temáticas de responsabilidad social- para todos los profesionales. Además, algunos de estos cursos se imparten paralelamente a las prácticas pedagógicas, lo que permite generar reflexión crítica y vinculación de la práctica con la teoría.

Tabla 10: Cursos de formación profesional general

\begin{tabular}{|l|l|}
\hline $\begin{array}{l}\text { Finlandia } \\
\text { Oulu University }\end{array}$ & $\begin{array}{l}\text { Segunda Lengua Oficial, Comunicación } \\
\text { Científica. }\end{array}$ \\
\hline $\begin{array}{l}\text { Finlandia } \\
\text { Helsinki University }\end{array}$ & $\begin{array}{l}\text { Escritura Científica; Lenguaje y Comunicación; } \\
\text { Segundo Idioma Nacional; Idioma Extranjero; } \\
\text { Información y Comunicación Tecnológicas. }\end{array}$ \\
\hline $\begin{array}{l}\text { Australia } \\
\text { Western Sydney University }\end{array}$ & $\begin{array}{l}\text { Segundo Idioma; Lingüística, Sociolingüística; } \\
\text { Bilingüismo y Biculturalismo. }\end{array}$ \\
\hline $\begin{array}{l}\text { Colombia } \\
\text { Universidad Pedagógica Nacional }\end{array}$ & $\begin{array}{l}\text { Comprensión y Producción de Textos I-II, } \\
\text { Idioma Extranjero I-IV (inglés), Laboratorio de } \\
\text { Lenguajes Artificiales. }\end{array}$ \\
\hline
\end{tabular}

Formación en investigación

Por último, en la mayoría de las universidades estudiadas se ofrece uno o más cursos de investigación. La formación en esta área es 
bastante variada; mientras en algunas se realizan uno o dos cursos, en la Universidad Pedagógica Nacional de Colombia, por ejemplo, se dictan seis seminarios de investigación como un continuo durante toda la carrera. Por otra parte, hay universidades que no incluyen estas asignaturas como es el caso de Universidad Complutense, Greenwich University, Auckland University. Esta variación curricular también depende de cada contexto, pues en varios casos las estudiantes pueden haber realizado estudios anteriores o proseguirán realizando otros estudios que posiblemente incorporen mayor formación en investigación. En la siguiente tabla se presentan los cursos ofrecidos con relación a la formación en investigación.

Tabla 11: Cursos de formación en investigación

\begin{tabular}{|l|l|}
\hline $\begin{array}{l}\text { Finlandia } \\
\text { Oulu University }\end{array}$ & Investigación \\
\hline $\begin{array}{l}\text { Finlandia } \\
\text { Helsinki University }\end{array}$ & $\begin{array}{l}\text { Filosofía de la Investigación y Metodología de la Investigación; } \\
\text { Investigación Cuantitativa; Investigación Cualitativa. }\end{array}$ \\
\hline $\begin{array}{l}\text { Inglaterra } \\
\text { Greenwich University }\end{array}$ & No se imparten. \\
\hline $\begin{array}{l}\text { España } \\
\text { Universidad Complutense }\end{array}$ & No se imparten. \\
\hline $\begin{array}{l}\text { Nueva Zelanda } \\
\text { Auckland University }\end{array}$ & No se imparten. \\
\hline $\begin{array}{l}\text { Australia } \\
\text { Melbourne University }\end{array}$ & Diseño de Proyecto I-II; Informe del Proyecto I-II \\
\hline $\begin{array}{l}\text { Australia } \\
\text { Western Sydney University }\end{array}$ & No se imparten. \\
\hline $\begin{array}{l}\text { Colombia } \\
\text { Universidad Pedagógica Nacional }\end{array}$ & Seminarios de investigación I-VI, Proyecto de grado I-IV. \\
\hline $\begin{array}{l}\text { Colombia } \\
\text { Universidad de Antioquia }\end{array}$ & $\begin{array}{l}\text { Ciencias Experimentales, Seminarios de investigación I-III, } \\
\text { Proyecto pedagógico I-III. }\end{array}$ \\
\hline
\end{tabular}

d.4) Cursos de formación profesional general Algunas universidades incluyen en sus mallas curriculares cursos de formación profesional general, como se muestra a continuación. 
Tabla 12: Cursos de desarrollo profesional

\begin{tabular}{|l|l|}
\hline $\begin{array}{l}\text { Finlandia } \\
\text { Oulu University }\end{array}$ & Desarrollo Profesional; Contextos Sociales y Ética Profesional. \\
\hline $\begin{array}{l}\text { Finlandia } \\
\text { Helsinki University }\end{array}$ & $\begin{array}{l}\text { Orientación a Estudios Universitarios y a la Profesión de } \\
\text { Educadora de Párvulos. }\end{array}$ \\
\hline $\begin{array}{l}\text { Nueva Zelanda } \\
\text { Auckland University }\end{array}$ & El Profesional Educador de Párvulos I-II \\
\hline $\begin{array}{l}\text { Australia } \\
\text { Melbourne University }\end{array}$ & $\begin{array}{l}\text { Orientación a la Profesión; Dimensiones de la Profesión; Roles } \\
\text { yelaciones Profesionales; Navegando en Distintos Contextos } \\
\text { Profesionales. }\end{array}$ \\
\hline $\begin{array}{l}\text { Australia } \\
\text { Western Sydney University }\end{array}$ & Profesional de la Educación de Párvulos. \\
\hline
\end{tabular}

Estas universidades incluyen una formación en competencias de lenguaje y comunicación, lo cual se plantea como un eje fundamental en la formación de estos profesionales. Ello incluye aspectos como lenguaje hablado, segundo idioma, expresión escrita, lectura académica, manejo de medios de comunicación y tecnología. Esto implica que le dan gran relevancia a estas competencias básicas, las cuales se deben seguir desarrollando posteriormente a la educación escolar; por lo tanto, se entiende que ésta no es suficiente para las exigencias de la formación o, eventualmente, que los niveles de entrada de los alumnos son muy desiguales.

\section{d.5) Prácticas pedagógicas y actividades de titulación}

La duración de las prácticas educativas es variada, fluctúa desde uno hasta diez semestres. En varios casos se realizan prácticas en distintos contextos (tipos de instituciones, niveles educativos), y también enfocadas hacia distintas temáticas (observación y planificación, evaluación, implementación curricular, trabajo de equipo, trabajo con familia, coordinación con otros servicios de la infancia). Generalmente, las prácticas se acompañan de cursos de reflexión, lo cual puede incluir sistemas de autoevaluación, uso de bitácora, supervisión de tutores y apoyo de "amigo crítico".

En cuanto a las actividades de titulación, además de la realización de las prácticas pedagógicas, en la mayoría de las carreras se debe realizar una tesis o investigación-acción junto con la práctica final. Aunque en algunas carreras, como en el caso de Greenwich University, Auckland University y Universidad Complutense, no se 
menciona la realización de una tesis, lo cual coincide con el hecho de que estas carreras no ofrecen cursos de investigación.

Tabla 13: Prácticas pedagógicas y actividades de titulación

\begin{tabular}{|c|c|}
\hline $\begin{array}{l}\text { Finlandia } \\
\text { Oulu University }\end{array}$ & $\begin{array}{l}\text { Dos prácticas semestrales. En la primera se trabaja la observación, } \\
\text { planificación, evaluación e implementación curricular. En la segunda } \\
\text { práctica, se incluye el trabajo con familia y otros servicios comunitarios, } \\
\text { y se genera un proceso de autoevaluación. Al final de la carrera se realiza } \\
\text { una tesis. }\end{array}$ \\
\hline $\begin{array}{l}\text { Finlandia } \\
\text { Helsinki University }\end{array}$ & $\begin{array}{l}\text { Tres prácticas. La primera práctica dura } 3 \text { semanas. La segunda práctica } \\
\text { dura } 6 \text { semanas y las últimas } 6 \text { semanas. } \\
\text { Se realiza una tesis y un examen al medio término de la carrera. }\end{array}$ \\
\hline $\begin{array}{l}\text { Inglaterra } \\
\text { Greenwich } \\
\text { University }\end{array}$ & Una práctica de 90 días, que se acompaña de un curso de Supervisión. \\
\hline $\begin{array}{l}\text { España } \\
\text { Universidad } \\
\text { Complutense }\end{array}$ & Una práctica de un semestre. \\
\hline \begin{tabular}{l|} 
Nueva Zelanda \\
Auckland University
\end{tabular} & $\begin{array}{l}\text { Tres prácticas, que duran en total } 5 \text { meses. Cada práctica se acompaña del } \\
\text { curso: Práctica en Educación de Párvulos. }\end{array}$ \\
\hline $\begin{array}{l}\text { Australia } \\
\text { Melbourne } \\
\text { University }\end{array}$ & $\begin{array}{l}\text { Tres prácticas. Primero, una práctica de } 45 \text { días en una escuela con el } \\
\text { primer ciclo básico. Segundo, una práctica de } 70 \text { días en un jardín infantil. } \\
\text { La práctica final es de } 5 \text { meses y medio, y puede ser en escuela o jardín } \\
\text { infantil. } \\
\text { En el último año se realiza el diseño de un proyecto educativo, y luego una } \\
\text { investigación de sus resultados. }\end{array}$ \\
\hline $\begin{array}{l}\text { Australia } \\
\text { Western Sydney } \\
\text { University }\end{array}$ & $\begin{array}{l}\text { Tres prácticas semestrales. La última se acompaña del curso: El Practicante } \\
\text { Reflexivo. }\end{array}$ \\
\hline $\begin{array}{l}\text { Colombia } \\
\text { Universidad } \\
\text { Pedagógica } \\
\text { Nacional }\end{array}$ & $\begin{array}{l}\text { Durante } 10 \text { semestres se realizan prácticas, acompañadas de seminarios } \\
\text { de investigación. Durante los últimos } 4 \text { semestres se realiza un proyecto } \\
\text { de grado. }\end{array}$ \\
\hline $\begin{array}{l}\text { Colombia } \\
\text { Universidad de } \\
\text { Antioquia }\end{array}$ & $\begin{array}{l}\text { Durante } 10 \text { semestres se realizan prácticas, acompañado con seminarios. } \\
\text { Los últimos } 3 \text { semestres se realiza un proyecto educativo. }\end{array}$ \\
\hline
\end{tabular}

Resultan interesantes las propuestas de ambas universidades colombianas, en las cuales se genera una línea continua de seminarios de investigación vinculados a las prácticas pedagógicas de los alumnos. Además, en estas universidades, como también en la Melbourne University, los egresados realizan el diseño de un proyecto educativo junto con una investigación-acción. Estas propuestas de formación podrían generar interesantes vínculos entre la teoría, la investigación y las prácticas pedagógicas. 


\section{Conclusiones. Tópicos de interés para pensar la formación en Educación Parvularia en Chile}

En este artículo se han analizado algunas tendencias internacionales de los programas de estudio para la formación de educadores de párvulos sobre la base del análisis de nueve universidades en distintos países. A partir de este examen podemos destacar las siguientes conclusiones:

- Las carreras universitarias tienden a ser más cortas que en Chile, pues la mayoría duran entre uno a tres años. La diferencia radica en que están pensadas para personas que ya poseen una profesión y experiencia de trabajo con niños o que continuarán con estudios posteriores. En este sentido, el perfil de ingreso se configura con estándares diferentes que en el caso chileno (que en la mayoría de los casos sólo exige el puntaje de la Prueba de Selección Universitaria (PSU) y las notas de enseñanza media) ${ }^{7}$. Además, se sugiere que los estudiantes combinen diferentes estudios y especializaciones por medio de licenciaturas, diplomados, minors y magísteres. Esto implica que los cursos que se imparten en las carreras de Educación Parvularia son principalmente del área de especialización. Los cursos de formación general son más bien escasos, pues esta formación se adquiere por medio de los diferentes estudios complementarios que realizan los estudiantes. En cuanto a los perfiles de egreso, es interesante considerar la propuesta de estándares de la profesión en Inglaterra. Estos generan orientaciones comunes para todas las universidades de un mismo país.

Sería interesante, en el ámbito de la formación en Chile, otorgar mayor importancia al perfil de ingreso del estudiante. Junto con la urgente necesidad de aumentar los puntajes de ingreso de la $\mathrm{PSU}^{8}$, se requiere diseñar instrumentos de selección que evalúen competencias básicas para futuros profesionales de la educación.

7 En el caso de Chile, existen carreras que contemplan una entrevista con el postulante; sin embargo, en el estudio no hallamos evidencia de que este tipo de instrumentos discriminen o seleccionen estudiantes.

8 En Chile el puntaje promedio de la PSU para ingresar a la carrera de Educación Parvularia es 450 puntos. Son muy escasas las instituciones que poseen puntajes de corte superior a los 600 puntos. 
- En los programas de estudio se destaca una formación holística e interdisciplinaria para estudiar temáticas de educación e infancia, en que se cruzan disciplinas como Filosofía, Historia, Psicología, Biología, Salud, Antropología/Sociología, además de Pedagogía y Psicología Evolutiva (las cuales han tenido mayor peso en las mallas chilenas). Niñas y niños son concebidos como sujetos integrales que requieren ser analizados y estudiados desde múltiples perspectivas. Los avances en materia de conocimiento humano y social, y los cambios permanentes propios de una sociedad que produce gran cantidad de información, obligan a los profesionales que trabajan con niños y niñas a tener una formación que les permita desarrollar visiones integrales de los grupos humanos.

Este punto es especialmente importante para el caso chileno. La evidencia internacional demuestra que el núcleo disciplinario de la formación está centrado en la infancia como un tema multidisciplinario. La tendencia en Chile es a favorecer ramos vinculados a la Psicología, descuidando la centralidad de otras perspectivas. Esto no ocurre en todas las carreras, pero sí marca una tendencia en la mayoría (García-Huidobro 2006).

- Frente a la discusión sobre el nivel de experticia del educador de párvulos, la tendencia que emerge es preparar a las estudiantes para trabajar con niños desde su nacimiento hasta los ocho años (aunque algunas carreras preparan hasta los 5 ó 6 años). La propuesta de extender la formación de párvulos hasta los ocho años pareciera interesante para favorecer la articulación con la educación básica, el trabajo con niveles heterogéneos, como también para que los educadores estén preparados para brindar experiencias desafiantes a niños con distintos niveles de desarrollo y aprendizaje. Esta tendencia muestra una propuesta de formar una experticia pedagógica en el ciclo completo de Educación de Párvulos. En dos universidades los estudiantes, posterior a una formación general en infancia, se especializan por etapas etarias, pero esta subdivisión se da en el ciclo de párvulos ( 0 a 6) y en el primer ciclo de educación básica (5 a 8), no entre el primer y segundo ciclo de la educación parvularia. 
- Desde la revisión de las mallas curriculares, aparecen áreas novedosas que no se trabajan de forma generalizada en las universidades e institutos de nuestro país, como el aprendizaje de una segunda lengua, educación multicultural, tecnología y medios audiovisuales, vida saludable y "alfabetización múltiple". Otro curso interesante que se ofrece en varias universidades es el de "diversidad e inclusión", que entrega un enfoque sociocultural de la infancia desde la perspectiva de los derechos de los niños y la equidad, respondiendo así a las diversidades étnicas, sociales, capacidades mentales y físicas, entre otros. En Chile ha predominado tradicionalmente una perspectiva de psicología evolutiva para comprender la infancia, por ello esta mirada desde la diversidad y los derechos de los niños sería un significativo aporte para la formación de educadores.

- Un énfasis importante en los cursos de trabajo con familia y comunidad es generar habilidades para el trabajo interdisciplinario. De esta forma el educador de párvulos no es un experto en trabajo con familia y servicio comunitario, sino que más bien debe saber cómo trabajar en redes con otros profesionales e instituciones. Este enfoque interdisciplinario ha sido escasamente trabajado en las universidades de nuestro país y además es un aspecto relevante de incorporar, puesto que las políticas y programas gubernamentales, como "Chile Crece Contigo", han promovido especialmente el trabajo en redes interinstitucionales.

- La administración y gestión institucional es una materia que raramente se imparte en las carreras analizadas y solamente de forma introductoria. Pareciera que, más bien, es un contenido de especialización que se trabaja en estudios posteriores.

- Las características de las prácticas pedagógicas son variadas. Sin embargo, existe una tendencia a ofrecer prácticas en diferentes momentos de la carrera, y que éstas se vinculen con proyectos de innovación, seminarios de investigación y/o talleres de desarrollo profesional. En este sentido, la tendencia es más o menos similar a lo que sucede en Chile.

Estas distintas propuestas y tendencias curriculares en diferentes países nos dan luces para repensar las tensiones y nudos críticos de 
la formación de educadores y educadoras de párvulos de nuestro país. Es de esperar que este estudio permita promover el diálogo y la discusión académica, con el fin de reflexionar desde el contexto chileno sobre cómo mejorar esta oferta formativa.

Las autoras agradecen a Solange Gorichón y Moira Lee quienes participaron de la investigación y realizaron comentarios a la sección del estudio internacional.

\section{Referencias bibliográficas}

Ávalos, Beatrice. (2003) La formación de los profesores y su desarrollo profesional. Prácticas innovadoras en busca de políticas. El caso de Chile. En: Cox, Cristián (Editor) Politicas educacionales en el cambio de siglo. La Reforma del sistema escolar de Chile. Santiago de Chile: Editorial Universitaria.

Brunner, José Joaquín. (2003) Politicas y mercados de educación superior: Necesidades de información. Santiago de Chile: Universidad Adolfo Ibáñez. Disponible en: http://mt.educarchile.cl/archives/Empleo_ capitulo\%25libro.pdf

Children's Workforce Development Council (2006) Early Years Professional National Standards. Disponible en: www.cwdcouncil.org.uk/pdf/ Early\%20Years/Draft_EYP_Standards_Aug_2006.pdf

García-Huidobro, Juan Eduardo. (2006) Formación inicial de educadoras(es) de párvulos en Chile. En Foco, Expansiva (80). 19/06/2006. http://www. expansivaudp.cl/en_foco/keywords.tpl?keyword=Educación

Kallós, D. (2003) Teachers and Teacher Education in Sweden Recent Developments. Disponible en: http://tntee.umu.se/archive/bologna2003.pdf

MINEDUC, CPEIP (2005) Construcción de Saberes Pedagógicos desde la implementación de la Reforma de Educación Parvularia. Santiago de Chile: MINEDUC.

Ministry of Social Affairs and Health (2004) Early Childhood Education and Care in Finland. Disponible en: http://www.stm.fi/Resource.phx/publishing/ store/2005/01/cd1106216815326/passthru.pdf

Moss, Peter. (2004) Fuerza laboral para la primera infancia en países 'Desarrollados': Estructuras y educación básicas. Nota de la UNESCO sobre las políticas de la Primera Infancia (27).

OECD (2006) Starting strong II. Early childhood and care. Paris: OECD.

Raczynski, Dagmar. (2006) Una política para la infancia temprana. Debates Sociales. Boletín Trimestral de Asesorías para el Desarrollo. 
Rojas, María Teresa; Gorichon, Solange; Falabella, Alejandra; Lee, Moira. Propuestas de mejoramiento para la formación de profesionales de educación parvularia. Informe de Investigación, Facultad de Educación, Universidad Alberto Hurtado 2008. Estudio encargado por el MINEDUC, JUNJI e INTEGRA. No publicado.

Training and Development Agency for Schools (2007) Professional Standards for Teachers Qualifies Teachers Status. Disponible en: http://www.tda.gov. uk/upload/resources/pdf/s/standards_qts.pdf Visitado: 7/08/2006

UNESCO (2007) The Training and Working Conditions of Preschool Teachers in France. Disponible en: http://unesdoc.unesco.org/images/0014/ 001499/149966E.pdf

University of Auckland (2007) Undergraduate Prospectus. Disponible en: http://www.education.auckland.ac.nz/uoa/education/about/news/ 2007/08/08prospectus.cfm

University of Helsinki (s/f) Curriculum for Kindergarten Teachers. Disponible en: http://www.helsinki.fi/sokla/english/Curriculum\%20for\%20kind ergarten\%20teacher\%20education.pdf

University of Oulu (2006) Information Package. Disponible en: http://www. oulu.fi/intl/exchange_students/ects/ktk/KASOPE06-07.pdf

University of Western Sydney (2005) Undergraduate Handbook. Disponible en: http://handbook.uws.edu.au/downloads/ 2005\%20Undergraduate\%20Handbook.pdf

Recibido: 17 de septiembre de 2008

Aceptado: 22 de octubre de 2008 